\title{
XX.-The Osmotic Pressure of Solutions of Sugar in Mixtures of Ethyl Alcohol and Water.
}

By Percival Smith Barlow.

Wren a copper ferrocyanide membrane is used with alcoholic solutions, no osmotic pressure is set up. This has been long known as the result of Tammann's work (Ann. Phys. Chem. Neue Folge, 1888, 34, 309), and has been further confirmed by the author. Other work (Phil. Mag., 1905, [vi], 10, 1) has also shown that this membrane is very sensitive to the presence of water. These results, obtained almost simultaneously, suggested the inquiry as to how far the presence of alcohol might modify the osmotic pressure of an aqueous solution of sugar if the experiment was arranged so that there were equal concentrations of water and alcohol on opposite sides of the membrane.

Cane sugar was used as the dissolved substance, since it is fairly soluble in a mixture of alcshol and water, and, being the substance employed in Pfeffer's experiments, which were the basis of the van't Hoff "gas" theory, it seemed the most suitable compound for the following work. Special conditions of experiment are employed. With the copper ferrocyanide membrane, the water of the solvent can alone produce an osmotic current, and of the pure liquids is the one which alone dissolves the sugar.

In preparing for each experiment, the clean cell was soaked for from one to two days in the solvent, this (unless otherwise stated) being a mixture of equal volumes of ethyl alcohol ("absolute") and water. The cell was then filled with the solution to be used in the experiment, and was again left for two or three days in the solvent. Fresh solution and solvent were used for the experiment itself. In order to avoid as far as possible any osmotic pressure which might arise from any inequality in the liquids themselves apart from the dissolved sugar, the solvent was always prepared beforehand in large quantity. The same liquid could then be used for soaking the cell, for making the solution, and for the outer liquid. Except in Experiment I, the cell stood in a glass bottle which was well corked. By means of a small mercury manometer through the cork, atmospheric pressure could be maintained above the outer liquid.

Experiment I. Strength of solution, 0.0075 normal. Theoretical pressure, $128 \mathrm{~mm}$. Initial pressure shown by the gauge was $39.5 \mathrm{~mm}$. After ten days this was $35 \mathrm{~mm}$., and was followed by a steady fall.

Experiment II. Strength of solution, 0.0117 normal. Theoretical pressure, $210 \mathrm{~mm}$. Initial pressure, $24 \mathrm{~mm}$. After thirteen days the pressure was $6.8 \mathrm{~mm}$. below atmospheric pressure. 
This result indicates a small outflow. It may be due to greater concentration of water inside the cell, in spite of the precautions taken. The membrane is certainly sensitive to small differences of concentration of water, but the difference in this case can only be very small, and should be more than counterbalanced by the sugar present.

Experiment III. Strength of solution, 0.027 normal. Theoretical pressure, $458 \mathrm{~mm}$. The cell was closed for five weeks. After that time a rise in pressure of $9 \mathrm{~mm}$. was shown.

If one can assume steady values after so long a time, this experiment indicates that as the strength of the sugar solution increases, the possibility of the osmotic pressure being shown also increases, and that there is a strength of solution the osmotic pressure of which is nullified by the alcohol present. Solutions below this strength show no osmotic pressure.

In Experiment IV, dextrose was the dissolved substance. The previous history of the cell used in this case is important, and is shortly as follows. The cell was washed in several changes of fresblyboiled distilled water for nine days. It was then used with an aqueous solution of dextrose. The cell was closed and under pressure for eleven days. After that time, the outer water was examined for dextrose by Fehling's solution; there was no reaction. Test cases showed that the solution was working well. This absence of sugar in the outer water is in agreement with the general behaviour of a copper ferrocyanide membrane, but is important evidence when one considers the result of the following experiment. Finally, the cell was washed for twelve days in distilled water, and then used in the ordinary way with the mixed solvent.

Experiment IV. Solution of dextrose: strength, 0.019 normal. Theoretical pressure, $325 \mathrm{~mm}$. The cell was closed for five weeks and then showed a pressure of $6 \mathrm{~mm}$.

The outer liquid was examined for dextrose, and a small but distinct precipitate of copper oxide was obtained. The membrane, however, had been proved (as above) to be impermeable to dextrose under greater internal pressure; it therefore appears as though the membrane ceased to be impermeable to the dextrose in the presence of alcohol. It will be necessary to consider this part of the experiment later. The actual osmotic effect is seen to be in agreement with those of the first three experiments.

Having found that, as the strengths of the solutions increased, the possibility of demonstrating the osmotic pressure also increased, it was natural to conclude that the greatest osmotic effect would be shown with saturated solutions. Whatever theoretical considerations we may apply to the grouping of the different molecules in these solutions, it will be found that there should be an inflow of water. If one 
considers the two liquids as aqueous solutions of alcohol, the inner solution also contains dissolved sugar. This, on the generally accepted theory, will necessitate an inflow, given a suitable membrane. The osmotic current always tends to set up conditions which oppose it ; in this case, this opposition is twofold. There is the increase of internal pressure and the diminution in the concentration of the water outside. Hence, with saturated solutions of sugar in this solvent, no very large pressure can be expected.

In the following experiments, the solutions were saturated. Excess of sugar was placed in the mixed solvent and left for three or four days, the mixture being shaken occasionally.

Experiment V. Solvent, 3 vols. of water to 1 vol. of alcohol. Saturated solution of cane sugar. After eight days, the osmotic pressure was $164 \mathrm{~mm}$. The cell was opened and closed several times; there was a steady rise in each case, showing altogether a large inflow. The gauge was an open one, and the highest pressure which could be shown on it was not large.

Experiment VI. Saturated solution of cane sugar in equal volumes of alcohol and water. After eight days, the pressure was $62 \mathrm{~mm}$.; after a month, it was $198 \mathrm{~mm}$.

Traube, as a result of his work on capillary constants of solutions (Phil. Mag., 1904, [vi], 8, 704), has put forward the theory that the osmotic current is caused by the difference of the surface tensions of the liquids separated by the membrane. In another connection, I have had occasion to show how previous work bears on this theory (Phil. Mag., 1905, [vi], 10, 11). The theory seems to demand the passage of the whole liquid ; with a membrane of copper ferrocyanide, the current is caused by the water alone. The theory, therefore, does not include a simple case. Moreover, the surface-tension theory neglects the part played by the membrane, and therefore does not take into account all the conditions of work.

In considering the function of the membrane, the author's work emphasises the importance of Nernst's researches on the absorptive action of the membrane. The part played by the membrane is itself a phenomenon of solution. One of the necessary conditions for an osmotic current is that the solvent (or one of the liquids) can be absorbed by the membrane.

Traube further argues, on lines difficult to follow, that in the presence of alcohol the membrane may be rendered permeable to the sugar or dissolved substance, to which it is impermeable in aqueous solution. The latter part of Experiment IV is in favour of this view. This breaking down of the impermeability of the membrane to sugar is especially difficult to understand in the light of the fact that the 
cells remain sound for further use. This isolated case affords no basis for inference, but shows that the point is worthy of further examination. If this temporary permeability does occur, it is probable that the alcohol forms a true solution with the sugar.

So far, the membrane used has been permeable to water only. Bladder is the only membrane known to the author which is permeable, in an osmotic sense, to alcohol and water; this was used with a brass cell consisting of two parts fitted together by flanges and screws, the prepared bladder being fixed between the flanges and two india-rubber rings. With such a cell, a cane-sugar solution in equal volumes of alcohol and water (theoretical pressure $=1240 \mathrm{~mm}$.), and far from being saturated, gave a rise in pressure of $140 \mathrm{~mm}$. in five days, a rate of increase greater than that in experiments with saturated solutions, even although bladder is imperfectly semipermeable. This result suggests that both parts of the solvent formed the osmotic current, as the previous knowledge of the membrane would lead one to expect. The selective action of the bladder would cause a larger inflow of water than of alcohol.

The last case is where the membrane allows the alcohol only to form the osmotic current. Such a membrane is obtained with gutta-percha tissue. This was used in the same way as the bladder in the type of cell just described. Three cells were used for these experiments, but two, after considerable care and time, gave no result. There seems reason for thinking that this is due to deterioration of the membrane, and not to the solutions being too weak. The last experiment is thought to be of interest because of the length of time over which it extends.

Experiment VII. Solvent: equal volumes of alcohol and water. Strength of solution, $0 \cdot 227$ normal. Theoretical pressure $=3850 \mathrm{~mm}$. Gutta-percha membrane.

After being closed for two months, the osmotic pressure was $48 \mathrm{~mm}$. In this experiment, the movement of the gauge can be no indication of the volume of the liquid which has crossed the membrane; the outward sag of the membrane necessitates a greater inflow than the same gauge-movement would indicate in the case of a copper ferrocyanide membrane. Alcohol is the inflowing liquid.

The ultimate object of osmotic research must be further knowledge of the internal conditions of solution. Incidentally there also arises the question of the cause of the current and the part played by the membrane. Previous reference to this makes further remark necessary. The cause of the current is found in the mutual potential energy of solution : that a current should flow depends on the ability of the membrane to dissolve the liquid. For mixed solvents, the liquid of the current depends on the selective action of the membrane. This 
method of explanation, in addition to being in agreement with experiment, brings osmotic phenomena into line with the principle that potential energy tends to a minimum.

On the matter of internal grouping of the molecules of the solution, not much can be said here. Recent work is all in support of "hydration" of the dissolved substance (whether ionised or not), and, more especially, work on non-aqueous solutions. It is still to be discovered why water dissolves sugar and alcohol does not, although one can quite truly say that there is some kind of attraction between sugar and water which does not exist between sugar and alcohol. In this paper, the question arises as to whether the alcohol has some of this "solution attraction" for the sugar after this has been brought into solution by the water. In the light of the last experiment, it is probable that there is "true" solution between the sugar and the alcohol. Here the osmotic current is one of alcohol, and in the usual osmotic sense the alcohol can be regarded as the solvent. If there is no true solution between the alcohol and the sugar, then the aggregation of the water and sugar molecules causes a greater concentration of free alcohol in the liquid containing the sugar than in the mixed solvent. Hence the flow of alcohol in the last experiment should have been outwards and not inwards. On the same assumption the van't Hoff "gas" theory would give the same result. Experiment is therefore in favour of the "gas" particles of the solution being aggregates of the three molecules: in other words, the sugar is "hydrated" by the water and the alcohol.

The difficulties of finding the osmotic pressures of these solutions have been mentioned. The selective action of the membrane causes inequalities in the concentrations of the liquids themselves. This might be avoided by adopting a method of applying pressure from the outside (see Lord Berkeley's paper, Proc. Roy. Soc., 1904, 73, 436), and so finishing the experiment before there could be any appreciable exchange of liquid. Without some such method, it appears to be futile to look for van't Hoff values for mixed solvents and their solutions.

These experiments were carried out at the Cavendish Laboratory; and, in conclusion, the writer thanks Professor J. J. Thomson for the kindness shown to him during the progress of the work. 